Document downloaded from:

http://hdl.handle.net/10251/56858

This paper must be cited as:

Torres, V.; Sánchez Losilla, N.; Etayo, D.; Ortuño Molinero, R.; Navarro-Cía, M.; Martínez Abietar, AJ.; Beruete, M. (2014). Compact Dual-Band Terahertz Quarter-Wave Plate Metasurface. IEEE Photonics Technology Letters. 26(16):1679-1682. doi:10.1109/LPT.2014.2330860.

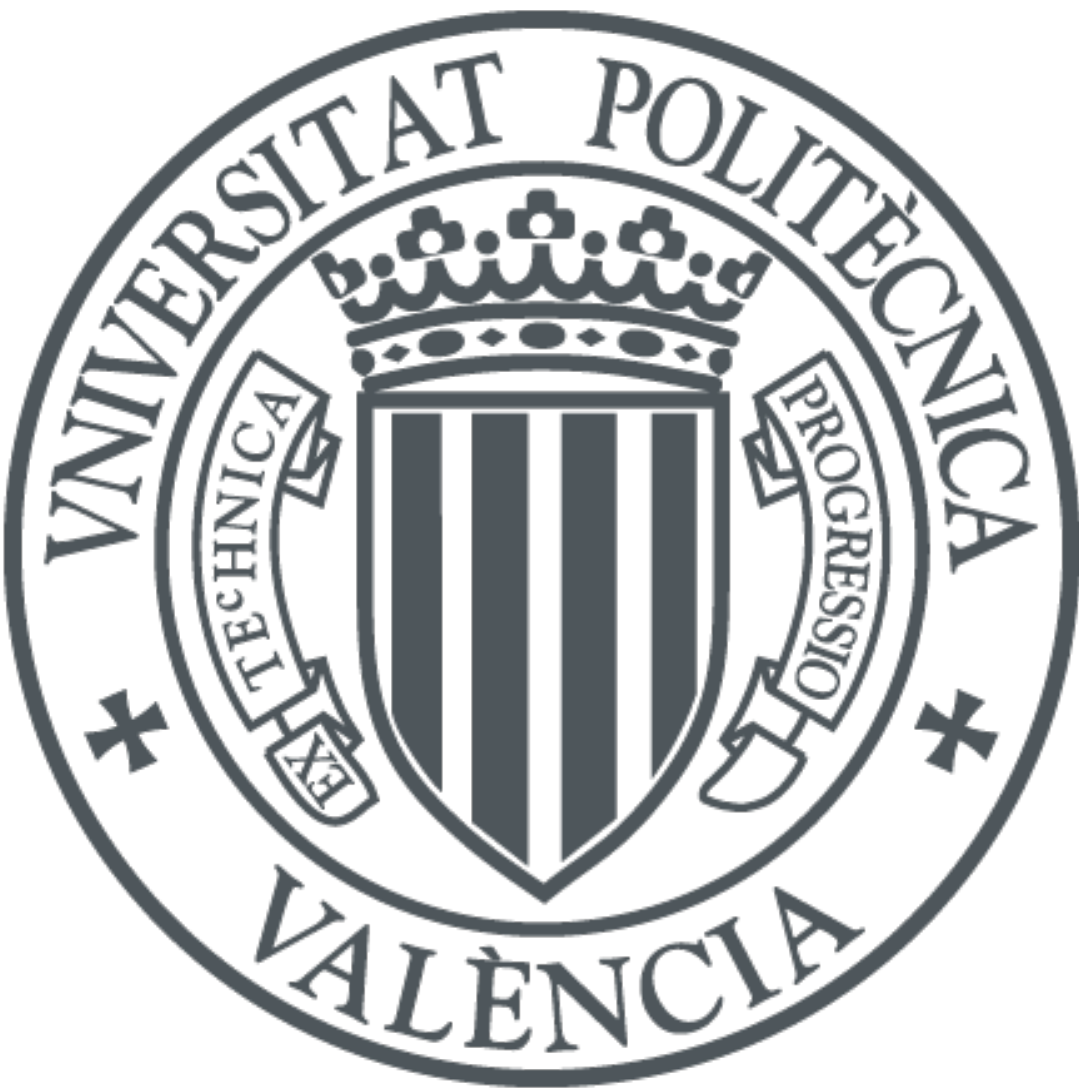

The final publication is available at

http://dx.doi.org/10.1109/LPT.2014.2330860

Copyright Institute of Electrical and Electronics Engineers (IEEE)

Additional Information

"(C) (C) 20xx IEEE. Personal use of this material is permitted. Permission from IEEE must be obtained for all other uses, in any current or future media, including reprinting/republishing this material for advertising or promotional purposes, creating new collective works, for resale or redistribution to servers or lists, or reuse of any copyrighted component of this work in other works." 


\title{
Compact Dual-Band Terahertz Quarter-Wave Plate Metasurface
}

\author{
Víctor Torres, Nuria Sánchez, David Etayo, Rubén Ortuño, Miguel Navarro-Cía, Member, IEEE, \\ Alejandro Martínez, Member, IEEE, and Miguel Beruete
}

\begin{abstract}
A dual-band quarter-wave plate based on a modified extraordinary transmission hole array is numerically analyzed and experimentally demonstrated at terahertz frequencies. To control independently orthogonal polarizations, the original square holes are connected with vertical slits and their lateral straight sides are replaced by meander lines. This smart design enables dual-band operation with unprecedented fractional bandwidths in a compact structure. Considering a flattening deviation lower than $40 \%$ of the optimum value, a fractional bandwidth of $53.8 \%$ and $3.8 \%$ is theoretically obtained $(16.8 \%$ and $2.9 \%$ in the experiment) at 1 and $2.2 \mathrm{THz}$, respectively. At these two frequencies, the structure is $0.13-\lambda$ and $0.29-\lambda$ thick, respectively. Given the compactness of the whole structure and the performance obtained, this quarter-wave plate is presented as a competitive device for the terahertz band.
\end{abstract}

Index Terms-Quarter-wave plate, terahertz applications, metasurface, extraordinary transmission, birefringence.

\section{INTRODUCTION}

D URING the last decades great efforts have been directed toward the development of terahertz (THz) technology due to the promising applications in imaging, semiconductor characterization and especially in biological and chemical sensing [1]. The development of $\mathrm{THz}(0.3-3 \mathrm{THz})$ timedomain spectroscopy has driven important advances in all these fields because it provides additional information to the widely used Fourier transform infrared spectroscopy [2]. This rapid progress has benefited from new generation and detection methods based on ultrafast pulses together with nonlinear crystals and photoconductive switches [3], [4]. Further

Manuscript received December 19, 2013; revised March 17, 2014; accepted June 11, 2014. Date of publication June 13, 2014; date of current version July 25, 2014. This work was supported by the Spanish Government through the Consolider Engineering Metamaterials under Contract CSD2008-00066 and Contract TEC2011-28664-C02.

V. Torres, R. Ortuño, and M. Beruete are with the TERALAB, Universidad Pública de Navarra, Pamplona 31006, Spain (e-mail: victor.torres@ unavarra.es; ruben.ortuño@unavarra.es; miguel.beruete@unavarra.es).

N. Sánchez and A. Martínez are with the Nanophotonics Technology Center, Universitat Politècnica de València, Valencia 46022, Spain (e-mail: nsanchez@ntc.upv.es; amartinez@ntc.upv.es).

D. Etayo is with the Antenna Group, Electric and Electronic Department, Universidad Pública de Navarra, Pamplona 31006, Spain (e-mail: david.etayo@unavarra.es).

M. Navarro-Cía is with the Optical and Semiconductor Devices Group, Centre for Terahertz Science and Engineering, Centre for Plasmonics and Metamaterials, Department of Electrical and Electronic Engineering, Imperial College London, London SW7 2AZ, U.K. (e-mail: m.navarro@imperial.ac.uk). work can be found in the development of passive quasioptical $\mathrm{THz}$ components such as lenses, filters or diffraction gratings [5], [6]. Despite this activity, there is still a lack of efficient devices, especially those able to work wideband or multiband that benefit from the broadband feature of the above mentioned sources and detectors.

Among all these quasi-optical devices, polarizers are unquestionably of great interest. Classical polarizing devices exploit birefringent materials [7], whereby orthogonal polarizations encounter different indices of refraction. When the phase difference between orthogonal components of equal magnitude is 90 degrees, conversion from linear to circular polarized waves and vice versa can be achieved. This type of polarizers is called quarter-wave plate (QWP). At $\mathrm{THz}$ frequencies, QWPs based on photonic crystals made of quartz, calcite or even regular paper, have been proposed [8]-[10]. However, all these structures alike grating-based QWPs [11] are relatively thick (more than one wavelength at the operation frequency). This drawback can be overcome by means of metasurfaces, which can show form birefringence by properly adjusting the geometry of the unit cell [12]-[15]. Nevertheless, in most cases, their resonant nature leads to narrow bands which are insufficient in most applications.

In this work, we propose a very compact birefringent structure that works as a QWP at two different frequencies, $1 \mathrm{THz}$ and 2.2 THz. At the highest frequency the proposed QWP operates at Fabry-Perot resonance, and thus, the fractional bandwidth is below $10 \%$. However, at the lowest frequency, a large fractional bandwidth beyond those obtained with frequency selective surfaces (1.7 times larger) is achieved as a result of the off-resonance operation.

\section{Design And Experimental Procedure}

As shown in the unit cell depicted in Fig. 1(a), the QWP proposed here is a modified extraordinary transmission hole array. The square holes composing the original array are connected with vertical slits and their lateral straight sides are substituted by meander lines. The structural parameters of the unit cell estimated from scanning electron microscopy (SEM) photographs are the following: $d_{\mathrm{y}}=120 \mu \mathrm{m}, d_{\mathrm{x}}=58 \mu \mathrm{m}$, slit width $w=4.9 \mu \mathrm{m}$, hole size $a_{\mathrm{x}}=41.3 \mu \mathrm{m}$ and $a_{\mathrm{y}}=47.9 \mu \mathrm{m}$, meander width $m=4.5 \mu \mathrm{m}$ and meander separation $s=7.475 \mu \mathrm{m}$. The metal pattern is gold ( $\mathrm{Au})$ with a thickness of $0.1 \mu \mathrm{m}$ and it is backed by a substrate of polypropylene (PP) of thickness $t=40 \mu \mathrm{m}$.

Two different approaches have been used in simulation to model the metal and the substrate. The initial optimization 

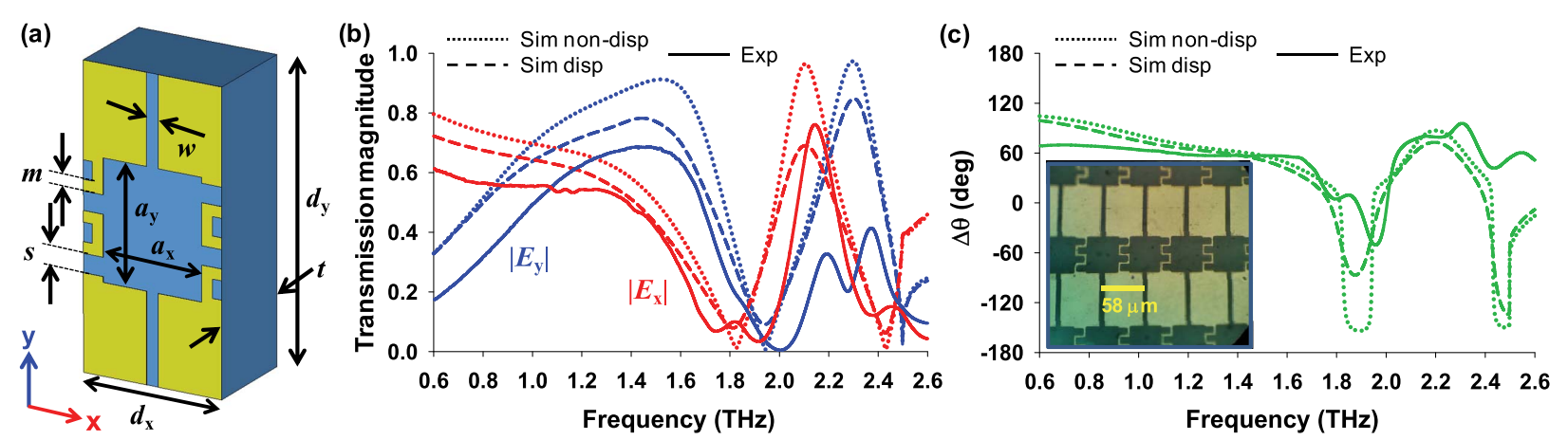

Fig. 1. (a) Unit cell. (b) Transmission magnitude and (c) phase difference between orthogonal components, when the structure is excited with a normal plane wave under $x$-polarization $\left|E_{\mathrm{x}}\right|$ and $y$-polarization $\left|E_{\mathrm{y}}\right|$. (inset) SEM photograph containing several periods of the prototype.

process of the structural parameters used a non-dispersive model where $\mathrm{Au}$ is assumed to have a finite electric conductivity equal to $4.561 \times 10^{7} \mathrm{~S} / \mathrm{m}$ laying over a PP layer with a constant permittivity $\varepsilon=2.22$ and loss tangent $\tan$ $\delta=0.001$ [16]. Under these assumptions the device operates as an ideal QWP, see dotted lines in Fig. 1(b,c). A parameter sensitivity analysis of this design is given in the supporting information. After the fabrication and measurement process, some deviation in the results was observed (see Section III for more details) and the previous simulation model was refined to explain the disagreements. A dispersive model was then used for both Au and PP: gold was modeled following a Drude model with plasma frequency $\omega_{\mathrm{p}}=8.67 \times 10^{16} \mathrm{rad} / \mathrm{s}$ and collision frequency $\omega_{\tau}=6.64 \times 10^{14} \mathrm{rad} / \mathrm{s}$ [17]; and for the PP dispersive a complex permittivity is extracted from measurements of a pristine metal-free PP sample. Real and imaginary parts of the permittivity are within the ranges $\varepsilon^{\prime} \in$ $\langle 2.21,2.42\rangle$ and $\varepsilon^{\prime \prime} \in\langle 0.1,0.6\rangle$, respectively. All simulation results have been obtained with the commercial software CST Microwave Studio ${ }^{\mathrm{TM}}$ using the frequency domain solver. The whole simulation is reduced to the analysis of a unit cell with Floquet boundary conditions under normal illumination. An adaptive tetrahedral mesh is set with a maximum step width of $0.3 \mu \mathrm{m}$ on the metal.

A SEM photograph of the prototype can be seen in the inset of Fig. 1(c). The fabrication process is based on wellknown "lift-off process", widely used in silicon photonics, adapted here to work with flexible substrates. The process begins by fixing the PP film to a 6 inch silicon wafer. After this, a layer of Microposit S1813 photoresist is spin-coated in an EVG 101 coater tool, dried for a minute at $110^{\circ} \mathrm{C}$ in a hot-plate (TEL Developer Marz VZ) and exposed in an EVG 620 mask aligner. The metallization is performed in an e-beam evaporator physical vapour deposition system (Pfeiffer Vacuum Classic 500 tool). The final step consists on the lift-off of the remaining photoresist with the metal excess, leaving the metal structure on the PP substrate.

The experiment was performed using a TPS Spectra 3000 from Teraview under nitrogen purging condition [18]. A hundred single measures with a $6 \mathrm{GHz}$ frequency step are taken to obtain an average representation of the transmittance. A plain sample of PP is used as reference.

\section{RESUlTS}

The electromagnetic response of our wave-plate metasurface under normal incidence is fully determined from the analysis of two orthogonal polarizations parallel to the principal axes, $x$ and $y$. Therefore, let us first study numerically the transmission properties of this metasurface for both orthogonal polarizations individually. The simulation results are plotted in Fig. 1(b,c) together with the experimental ones to facilitate the comparison. At low frequencies, under $y$-polarization (electric field parallel to the $y$-axis), transmission magnitude $\left|E_{\mathrm{y}}\right|$ tends to zero while under $x$-polarization (electric field parallel to the $x$-axis), transmission $\left|E_{\mathrm{x}}\right|$ tends to one (Fig. 1(b)). This is due to the aperture shape as it will be explained subsequently. In the non-dispersive case, both components cross at $f_{0}=1 \mathrm{THz}$, when the transmission magnitude is equal to $\left|E_{x}\left(f_{0}\right)\right|=\left|E_{y}\left(f_{0}\right)\right|=1 / \sqrt{2}$, i.e. transmittance is $50 \%$. Moreover, a phase difference of $\Delta \theta\left(f_{0}\right)=85.3 \mathrm{deg}$ is obtained (Fig. 1(c)) with a negligible cross polar component (i.e. output $E_{\mathrm{x}}$ when the input is $E_{\mathrm{y}}$ and vice versa) of 0.03 . Additional peaks rise for both components at $2.1 \mathrm{THz}\left(\left|E_{\mathrm{x}}\right|\right)$ and $2.3 \mathrm{THz}\left(\left|E_{\mathrm{y}}\right|\right)$ corresponding to a Fabry-Perot resonance between the metallic- and air-dielectric interfaces [19], [20]. At $f_{1}=2.2 \mathrm{THz}$, the transmission of both components is again equal $\left|E_{x}\left(f_{1}\right)\right|=\left|E_{y}\left(f_{1}\right)\right|=1 / \sqrt{2}$, the phase difference is $\Delta \theta\left(f_{1}\right)=87.1 \mathrm{deg}$ and the cross polar component 0.02 . Therefore, an almost ideal QWP is achieved in both bands.

Attending now to the experimental results, they clearly resemble the simulation ones. The number of peaks and dips accords with the simulation as well as the low frequency response. However, the high frequency peak for $y$-polarization is split into two probably due to fabrication imperfections which produce an undesired resonance. First and second band are centered at $1.07 \mathrm{THz}$ and $2.29 \mathrm{THz}$, respectively in the measured structure. The magnitude of the transmission is equal to 0.55 and 0.23 , respectively, somewhat below the simulated values, suggesting that experimental losses are higher than expected. Notice that the QWP response is slightly blueshifted, specifically $7 \%$ for $f_{0}$ and $4 \%$ for $f_{1}$. Observing the phase difference, good agreement is also obtained over the whole frequency range. Major differences are observed around the dips at $1.9 \mathrm{THz}$ and $2.5 \mathrm{THz}$, where the transmissions are 
TABLE I

SUMMARY OF THE QWP PERFORMANCE

\begin{tabular}{cccc}
\hline \hline & Sim. non-disp. & Sim. disp. & Exp. \\
\hline$f_{0}$ & $1 \mathrm{THz}$ & $1.01 \mathrm{THz}$ & $1.07 \mathrm{THz}$ \\
$\left|\mathrm{E}_{\mathrm{x}, \mathrm{y}}\right|\left(f_{0}\right)$ & 0.7 & 0.64 & 0.55 \\
$\Delta \theta\left(f_{0}\right)$ & $85.3 \mathrm{deg}$ & 74.2 & $62.6 \mathrm{deg}$ \\
$\mathrm{BW}\left(f_{0}\right)$ & $52.08 \%$ & $53.85 \%$ & $16.77 \%$ \\
\hline$f_{1}$ & $2.2 \mathrm{THz}$ & 2.18 & $2.29 \mathrm{THz}$ \\
$\left|\mathrm{E}_{\mathrm{x}, \mathrm{y}}\right|\left(f_{1}\right)$ & 0.7 & 0.59 & 0.23 \\
$\Delta \theta\left(f_{1}\right)$ & $87.1 \mathrm{deg}$ & $72.6 \mathrm{deg}$ & $95.3 \mathrm{deg}$ \\
$\mathrm{BW}\left(f_{1}\right)$ & $4.32 \%$ & $3.78 \%$ & $2.90 \%$ \\
\hline \hline
\end{tabular}

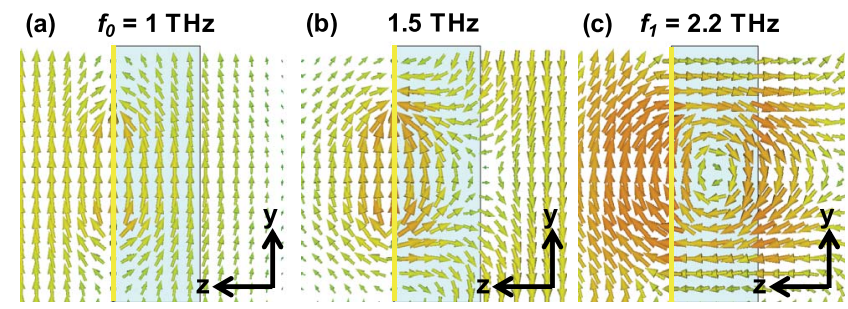

Fig. 2. Electric field representation on the $y z$-plane at the middle of the unit cell when the structure is excited with $y$-polarization.

zero and therefore fast phase changes might be expected [21] which are hardly measurable with the set up used. However, as they are located out of the bands of interest, they do not affect the performance. Specifically, at the center of the operation bands, a phase difference of $62.6 \mathrm{deg}$ and $95.1 \mathrm{deg}$ is measured for the lowest and the highest frequency, respectively.

Comparing the experimental results with the more realistic dispersive simulation, better agreement is obtained. Transmission values are closer because losses are better estimated and the frequency shift is partially predicted due to the dispersive nature of the model. Also the phase difference shows better prediction and the abrupt phase changes are softer, as they are in the experiment. A summary of the simulation and experimental results is shown in Table I where differences between simulation and experiment may be attributed to: (i) fabrication and material tolerances and (ii) misalignments during the experimental procedure (due to the flexibility of the structure, a flat and stable placement on the sample holder is difficult). The bandwidth (BW) is defined according to the flattening of the polarization state, as explained later.

The off-resonant and resonant feature of both bands is corroborated in Fig. 2 where the electric field under $y$-polarization is shown. It is observed at $f_{0}$ (Fig. 2(a)) how the electric field distribution shows a weak resonance condition in comparison with the field at the resonant frequency $1.5 \mathrm{THz}$ (Fig. 2(b)), where it is mainly confined at the hole of the metallic layer. On the contrary, at $f_{1}$ (Fig. 2(c)), the electric field is extended to the whole substrate and the Fabry-Perot resonance is clearly noticed. Similar results are obtained for $x$-polarization where the field confinement in the aperture and the Fabry-Perot resonance are observed as well.

As shown, the precise combination of holes and slits yields in the desired magnitude and phase retardation between $x$ - and $y$-polarization [22]. This can be easily explained from a circuital point of view. Under $x$-polarization, the electromagnetic wave effectively sees a modified slit array which behaves as a capacitance [23]. Hence the transmission tends to one at low frequency. On the other hand, under $y$-polarization, the wave sees essentially a modified hole array which is known to have an inductive behavior [24] and therefore, the transmission at low frequency tends to zero. This performance, based on complementary capacitive and inductive response, allows controlling the polarization state to achieve the desired 90 deg phase retardation between orthogonal components, as traditionally has been done, for instance, in classic meanderline polarizers [25]. However, the latter usually need a multilayer configuration to have high transmittance (at least two layers for similar performance).

Unlike a regular slit array, which shows a wide band response before the first order of diffraction (which is in fact our zone of interest), a regular hole array exhibits an extraordinary transmission resonance that it is narrow band by nature [26]. In order to achieve a wider band response, meander-lines are introduced between adjacent holes. It has already been proved numerically and experimentally [27], [28] that the total inductance and the bandwidth of the extraordinary transmission are increased with this technique. Notice that, in this work, the meander-line acts just as an inductance addition for the $y$-polarization, so it is not the source of the capacitance as in classical meander-line QWP [13], [25]. This capacitance is achieved for the $x$-polarization by means of the vertical slits.

In general, the electric field, at each propagation plane, is the superposition of the fields of both components, which in phasor notation is written as [19]:

$$
\vec{E}=\hat{x}\left|E_{x}\right|+\hat{y}\left|E_{y}\right| e^{j \Delta \theta}
$$

which, assuming that the phase is deterministic, represents an elliptical polarized wave in the most general case

From here, it is straightforward to calculate the major semiaxis $a$ and the minor semi-axis $b$ of the polarization ellipse by simply taking $a=\max |\vec{E}|$ and $b=\min |\vec{E}|$. The polarization state can be quantified by the flattening parameter defined as:

$$
\text { flatt }=1-(b / a)
$$

which indicates the ellipticity of the polarization, where $0 \leq$ flatt $\leq 1$, flatt $=0$ for circular polarization and flatt $=1$ for linear polarization. Considering flatt $<0.4$ as an acceptable approximation to circular polarization, our design has a bandwidth of $\mathrm{BW}\left(f_{0}\right)=0.47 \mathrm{THz}(16.77 \%)$ and $\mathrm{BW}\left(f_{1}\right)=0.07 \mathrm{THz}(2.9 \%)$ in a structure as compact as $\lambda / 7.5$ and $\lambda / 3.4$ for the $1 \mathrm{THz}$ and $2.2 \mathrm{THz}$ band, respectively. However, according to the simulation, a bandwidth around $50 \%$ and $4 \%$ might be achieved. In Fig. 3(a) it is shown the flattening for the whole frequency range. The measured flattening agrees well with the simulated one and it is observed how, where the bands are located, the flattening reaches the lower values. Around $1.9 \mathrm{THz}$ a dip is observed in the dispersive simulation but its transmission magnitude is very low, see Fig. 1(b). Shadowed areas indicate the bandwidths according to flatt $<0.4$. The polarization state of the transmitted wave 


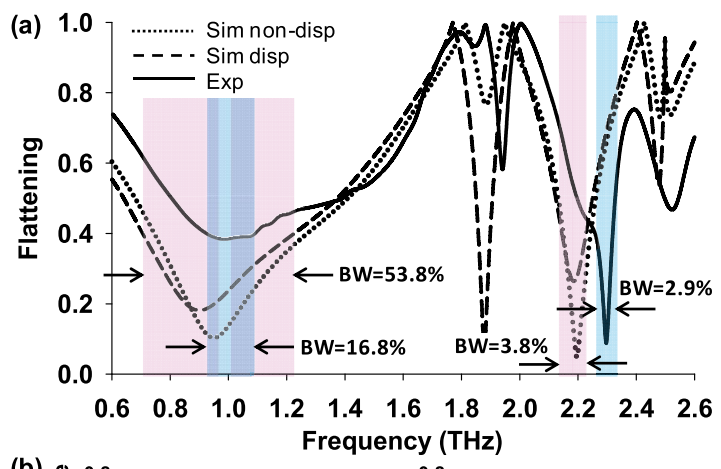

(b)

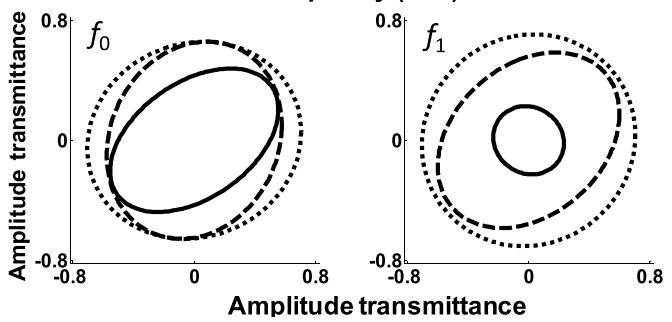

Fig. 3. (a) Flattening of the polarization state for the transmitted wave. Shadowed areas represent the operational bandwidths. (b) Polarization state at the optimum frequencies.

at the optimum frequencies (for the lowest flattening value) in each case is depicted in Fig. 3(b). Notice an acceptable experimental polarization state which is even better than the dispersive simulation at $f_{1}$ (although it is penalized by a lower amplitude transmittance) and the almost perfect circular shape for the ideal simulation.

\section{CONCLuSions}

In conclusion, a very thin QWP working at two different bands in the terahertz-gap is presented. The form birefringence is achieved by means of the excitation of a complementary capacitive and inductive response which is obtained through a single layer extraordinary transmission modified hole array backed with a PP substrate. Wide and narrow operation bandwidths of $16.77 \%$ and $2.90 \%$ at $1.1 \mathrm{THz}$ and $2.3 \mathrm{THz}$, respectively, are obtained after experimental results in a really thin structure of $0.29-\lambda$ thickness. Numerical analysis also shows that better performance can be achieved up to fractional bandwidth of $53.85 \%$ and $3.78 \%$. This work shows the potential that extraordinary transmission structures have for wave-plates competitive with those available in the literature (a comparison is given in the supporting information) and sets the bases for new $\mathrm{THz}$ quasi-optical polarizing devices.

\section{REFERENCES}

[1] Y.-S. Lee, Principles of Terahertz Science and Technology. New York, NY, USA: Springer-Verlag, 2009.

[2] S. L. Dexheimer, Terahertz Spectroscopy: Principles and Applications. Boca Raton, FL, USA: CRC Press, 2007.
[3] Q. Wu and X.-C. Zhang, "Ultrafast electro-optic field sensors," Appl. Phys. Lett., vol. 68, no. 12, pp. 1604-1606, Mar. 1996.

[4] L. Xu, X.-C. Zhang, and D. H. Auston, "Terahertz beam generation by femtosecond optical pulses in electro-optic materials," Appl. Phys. Lett., vol. 61, no. 15, pp. 1784-1786, Oct. 1992.

[5] V. Torres et al., "Terahertz epsilon-near-zero graded-index lens," Opt. Exp., vol. 21, no. 7, pp. 9156-9166, Apr. 2013.

[6] Y. Ma, A. Khalid, T. D. Drysdale, and D. R. S. Cumming, "Direct fabrication of terahertz optical devices on low-absorption polymer substrates," Opt. Lett., vol. 34, no. 10, pp. 1555-1557, May 2009.

[7] C. Jördens, M. Scheller, M. Wichmann, M. Mikulics, K. Wiesauer, and M. Koch, "Terahertz birefringence for orientation analysis," Appl. Opt., vol. 48, no. 11, pp. 2037-2044, Apr. 2009.

[8] J.-B. Masson and G. Gallot, "Terahertz achromatic quarter-wave plate," Opt. Lett., vol. 31, no. 2, pp. 265-267, 2006.

[9] P. Hariharan and P. E. Ciddor, "Superachromatic circular polarizer," Meas. Sci. Technol., vol. 11, no. 11, pp. N117-N118, Nov. 2000.

[10] B. Scherger, M. Scheller, N. Vieweg, S. T. Cundiff, and M. Koch, "Paper terahertz wave plates," Opt. Exp., vol. 19, no. 25, pp. 24884-24889, Dec. 2011.

[11] S. C. Saha, Y. Ma, J. P. Grant, A. Khalid, and D. R. S. Cumming, "Lowloss terahertz artificial dielectric birefringent quarter-wave plates," IEEE Photon. Technol. Lett., vol. 22, no. 2, pp. 79-81, Jan. 15, 2010.

[12] M. Navarro-Cia, P. Rodriguez-Ulibarri, V. Torres, and M. Beruete, "Quarter-wave plate based on dielectric-enabled extraordinary resonant transmission," IEEE Photon. Technol. Lett., vol. 24, no. 11, pp. 945-947, Jun. 1, 2012.

[13] A. C. Strikwerda, K. Fan, H. Tao, D. V. Pilon, X. Zhang, and R. D. Averitt, "Comparison of birefringent electric split-ring resonator and meanderline," Opt. Exp., vol. 17, no. 1, pp. 136-149, Dec. 2008.

[14] P. Weis, O. Paul, C. Imhof, R. Beigang, and M. Rahm, "Strongly birefringent metamaterials as negative index terahertz wave plates," Appl. Phys. Lett., vol. 95, no. 17, p. 171104, Oct. 2009.

[15] N. Yu, F. Aieta, P. Genevet, M. A. Kats, Z. Gaburro, and F. Capasso, "A broadband, background-free quarter-wave plate based on plasmonic metasurfaces," Nano Lett., vol. 12, no. 12, pp. 6328-6333, Dec. 2012.

[16] S. A. Kuznetsov et al., "Regular and anomalous extraordinary optical transmission at the THz-gap," Opt. Exp., vol. 17, no. 14, pp. 11730-11738, Jul. 2009.

[17] P. B. Johnson and R. Christy, "Optical constants of the noble metals," Phys. Rev. B, vol. 6, no. 12, pp. 4370-4379, 1972.

[18] (2014, Jul. 6). Teraview-Terahertz Products. [Online]. Available: http://www.teraview.com

[19] S. Ramo, J. R. Whinnery, and T. Van Duzer, Fields and Waves in Communication Electronics. Hoboken, NJ, USA: Wiley, 1994.

[20] R. Ortuño, "Multiple extraordinary optical transmission peaks from evanescent coupling in perforated metal plates surrounded by dielectrics," Opt. Exp., vol. 18, no. 8, pp. 7893-7898, 2010.

[21] M. Navarro-Cía, M. Beruete, F. Falcone, M. Sorolla, and V. Lomakin, "Negative group delay through subwavelength hole arrays," Phys. Rev. $B$, vol. 84, no. 7, p. 075151, Aug. 2011.

[22] M. Beruete, M. Sorolla, M. Navarro-Cía, and I. Campillo, "Polarized left-handed extraordinary optical transmission of subterahertz waves," Opt. Exp., vol. 15, no. 13, pp. 8125-8134, 2007.

[23] N. Marcuvitz, Waveguide Handbook. London, U.K.: McGraw-Hill, 1951.

[24] F. Medina, F. Mesa, and R. Marqués, "Extraordinary transmission through arrays of electrically small holes from a circuit theory perspective," IEEE Trans. Microw. Theory Techn., vol. 56, no. 12, pp. 3108-3120, Dec. 2008.

[25] B. A. Munk, Frequency Selective Surfaces. Hoboken, NJ, USA: Wiley, 2000.

[26] F. J. Garcia-Vidal, T. W. Ebbesen, and L. Kuipers, "Light passing through subwavelength apertures," Rev. Modern Phys., vol. 82, no. 1 , pp. 729-787, Mar. 2010.

[27] M. Beruete, M. Navarro-Cía, V. Torres, and M. Sorolla, "Redshifting extraordinary transmission by simple inductance addition," Phys. Rev. $B$, vol. 84, no. 7, pp. 1-5, Aug. 2011.

[28] V. Torres et al., "Mid-infrared plasmonic inductors: Enhancing inductance with meandering lines," Sci. Rep., vol. 4, no. 3592, pp. 1-5, Jan. 2014. 